\title{
Second invited commentary to the letter by Jan Lambrecht
}

\author{
P. K. Amid • D. C. Chen
}

Received: 12 March 2011/Accepted: 1 April 2011/Published online: 27 April 2011

(C) Springer-Verlag 2011

\section{Dear Editor,}

We appreciate Dr. Lambrecht's clarification [1] that his proposed coefficient for comparison of the ideal mesh tissue overlap between studies of ventral hernia repairs is limited to laparoscopic IPOM.

Restriction of defect size for IPOM repair is a controversial matter, and we leave it to the judgment and experience of the readers of Hernia. For large defects, we at UCLA, in common with many hernia specialists, prefer the compartment separation technique, particularly with an endoscopic relaxing incision to avoid the morbidity associated with skin flaps.

The size of mesh needed to achieve optimal overlap in these larger defects makes laparoscopic IPOM repair less than ideal. Additionally, the patient will continue to have an adynamic abdominal wall due to the persistent lateralization of the abdominal musculature. By combining endoscopic bilateral myofascial advancent flaps or "component separation" with a the retromuscular Rives Stoppa repair, the mesh is placed entirely extraperitoneally, the recurrence rates are historically low due to the placement of the mesh behind the rectus muscle, and the abdominal musculature is allowed to return to its natural position thus restoring the dynamic nature of the abdominal wall in a tension-free manner. Dr. Lambrecht's coefficient is a commendable effort towards establishing a uniform method of comparing ideal mesh tissue overlap in laparoscopic IPOM. While it does not apply to all techniques, hernia sizes, and locations, it may serve as a foundation for further study and uniform comparison of the traditionally accepted indications for laparoscopic repair and to identify limitations regarding defect size in a systematic and quantifiable fashion.

Thank you for this opportunity to respond to Dr. Lambrecht's letter.

\section{Reference}

1. Amid PK Invited commentary to letter by Jan Lambrecht. Hernia. doi:10.1007/s10029-011-0818-9
P. K. Amid ( $\square)$

David Geffen School of Medicine at UCLA,

Lichtenstein Hernia Institute at UCLA, Los Angeles, CA, USA

e-mail: pamid@onemain.com

D. C. Chen

UCLA Division of General Surgery, Los Angeles, CA, USA 